\title{
Grand Challenges in Planetary Nebulae Studies: Binary Evolution and MHD
}

\author{
Adam Frank \\ Department of Physics and Astronomy, University of Rochester, \\ Rochester, NY 14627, USA \\ email: afrank@pas.rochester.edu
}

\begin{abstract}
We review work on the evolution of planetary nebulae and proto-planetaries via magneto-rotational mechanisms showing that a dynamo generated magnetic field can produce the energy and momentum needed to drive pPN and PNe outflows. Angular momentum considerations lead to the conclusion that single stars can not support strong fields for long times. Thus we take the working hypothesis that most PN may form via binary stars. We propose that the grand challenge for PN studies is fully understanding the diverse physical processes at work in binary late stage evolution including the development of disks, fields and outflows.
\end{abstract}

Keywords. Planetary nebulae: general, stars: mass loss, magnetic fields

\section{Introduction: The State of PNe Research}

In recent years the field of Planetary Nebula research has approached something of a cross-roads. The impressive success of radiation transfer, micro-physical modeling of nebular plasma conditions and 1-D radiation hydro-dynamic models applicable to spherical nebula have provided confidence that a fairly complete understanding of parameters within the nebula had been achieved. This success has also raised the question of the direction for the field. In the absence of an overarching science question, the field of PNe studies is in danger of becoming eclipsed by other domains of interest in astronomy which appear to have more visible and compelling frontiers.

The impression that PNe is a study of "questions mostly answered" is wrong however. The last decade has also shown that, in spite of great progress, our understanding of both meso-physical (Huggins, these proceedings) and macro-physical (Balick, these proceedings) nebular characteristics remain far from certain. High resolution images from platforms like the HST have shown that we still lack a well tested model which can explain the shapes of $\mathrm{PNe}$ and link them, in a consistent way, to the evolution of the central star. In this contribution I review some outstanding issues in pPNe and PNe shaping and suggest how these provide us with a new way of looking at PNe studies. The perspective I advocate is that the frontiers of PNe studies are broad and, most importantly, provide links to some of the most pressing questions in a variety of other astronomical environments.

\subsection{Arguments for MHD Launching and Collimation}

The need for magnetic fields to aid in shaping the nebula has been discussed before (Balick \& Frank 2002). The work of Garcia-Segura, Lopez and others (Garcia-Segura et al. 1999) has demonstrated that including magnetic fields on nebular scales $R>10^{16} \mathrm{~cm}$ leads to a wide variety of morphologies including well collimated jets and point symmetry. What these models do not address however is the launching of the wind. 
The work of Bujarrabal, Alcolea, Sanchez-Contreras and others makes "weak field" models untenable and provides one of the most important challenges to PNe studies. It also offers a cogent direction forward for the future. In Bujarrabal et al. (2001) the momentum and energy budgets for both AGB and pPN outflows where derived. Their conclusion was that while AGB outflows could be satisfactorily explained via radiation driving, pPNe outflows showed momentum "excesses" that where $10^{3}$ or higher. Such large values of the ratio of outflow momentum to what could be supplied by photons, $P=L / c$, could not be accounted for via multiple scattering. Thus another source of energy is needed to produce the wind. Bujarrabal et al. (2001) also claimed that the acceleration timescales for the outflows should be short $(t<1000 \mathrm{y})$. Thus pPN, and subsequent, PNe may initially form via an outburst or explosion rather than a continuous wind.

It also noteworthy that the statistics of pPN and young PN morphologies appear to differ dramatically from fully developed PNe. The samples of pPNe of Bujarrabal and collaborators and of young PNe (Sahai \& Trauger 1998) are both dominated by strongly bipolar outflows. It remains to be seen if this is a observational bias or not. If such a distinction between older and younger population maintains after further study, it will indicate that the early shaping of PNe occurs via forces which differ as the nebula matures.

\section{Magneto-Rotational Launch (MRL) Models}

In magneto-rotational outflow theory the rotational energy of a central gravitating source is tapped via an embedded magnetic field to launch material past the escape speed and create a wind. At some distance the magnetic stresses collimate the wind into a narrow jet or broader bipolar outflow. In works by Blackman, Frank and collaborators MRL models have been explored in detail. In what follows we briefly review these works.

Disk+Star Model: In Blackman, Frank \& Welch (2001a) a model was proposed which assumed a disk forming via disruption of a binary. In this model both the disk and the rotating core of the central star produce a wind via magneto-rotational processes. The key expression related the wind mechanical luminosity to the magnetic luminosity of the rotator (star or disk)

$$
L_{w} \approx \dot{M}_{w} v_{w}^{2} \sim L_{m}=\int(\mathbf{E} \times \mathbf{B}) \cdot d \mathbf{S} \sim \int_{R_{i}}^{R_{o}}(\Omega R) B_{p} B_{\phi} R d R,
$$

These models were successful in outlining the mechanisms and rough time-dependencies of the wind launching and established a basis for a MRL paradigm for pPNe.

Disk Only Models: In Frank \& Blackman (2004) a version of MRL launching where centrifugal forces along the poloidal component of the field dominate initially (a "Fling") was applied to pPNe and PNe disks. Here scaling relations were derived from equations for such magneto-centrifugal launching. From these relations it was shown that the energy and momentum budgets for pPN could be recovered. In addition conditions typical of a mature PN could also be supported via magneto-centrifugal wind launching.

Single Star Models: In Blackman et al. (2001b) a single star model was proposed for the development of collimated pPN outflows. This work used a model for an initially rotating $3 M_{\odot}$ star that was evolved to the tip of the AGB (Kawaler-private communication) with the assumption of angular conservation on shells. An interface dynamo model was then applied to the resulting rotation profile $\Omega(R)_{*}$. The results showed that a dynamo of sufficient strength was produced to power an impulsive outflow with energy matching what was found in pPNe. In this model it was assumed that the outflow would be initiated as overlaying material was peeled away by the AGB wind. 


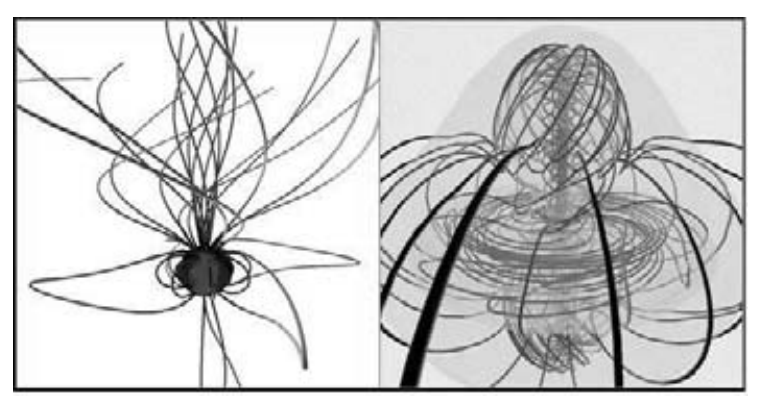

Figure 1. 3-D rendering of field lines and density in magnetic explosion models. Left: central regions. Right: overall morphology. Note dominance of toroidal fields.

More recently Matt, Frank \& Blackman (2006) have explored the process of wind launching from an exposed, rotating stellar core in more detail. Their simulations tracked a core with an initially dipole or split monopole field. The core was surrounded by an envelope in hydrostatic equilibrium which was not rotating. The differential rotation between the core and the envelope converted poloidal field $B_{p}$ into toroidal field $B_{\phi}$. As the field winds up magnetic pressure gradients $\sim \nabla B_{\phi}^{2}$ eventually drive the envelope beyond the local escape speed (a "Spring" model). We note that the key parameter which determined the launch of the outflow was $\chi=\left(v_{a} v_{r o t}\right)^{1 / 2} / v_{e s c}$. We found that $\chi>0.2$ should be the limit for initiating the explosion.

The outflow which forms from such a configuration was strongly collimated, though an equatorial outflow could also form depending on the field configuration (Fig. 1). It is important to note that the outflow is transient producing a kinetic energy dominated "cap" riding on a Poynting flux dominated jet. Such Poynting flux outflows are the subject of considerable interest in other domains such as GRBs. Thus the outflow forms more of a magnetic explosion than a continuous flow. Such conditions may be well suited to describing pPNe. In a recent set of simulations (Cunningham \& Frank 2006, in preparation) we have attempted to model the pPNe, CRL 618 as a collection of bullets impulsively launched from the source. Our initial results show such a model is effective at reproducing the gross morphology and kinematics of the nebula (Fig. 2).

\section{Binaries, Dynamos Models and the Future}

The importance of binaries in PNe shaping continues to be a subject of debate. For many years Soker (and Livio) has argued that angular momentum exchange via some form of companion would play a strong role in PNe evolution (i.e. Soker \& Rappaport 2000: also see De Marco these proceedings). Clearly all models involving accretion disks require the presence of a binary companion. What was not initially appreciated however was the need for a binary to maintain conditions for a strong dynamo in an AGB star. Soker initially made this point and our own work supports this conclusion. The problem can be couched in terms of energy considerations as each dynamo cycle involves the removal of magnetic energy. Thus rotational energy is continually lost to magnetic field which must then be regenerated. Recently Nordhaus \& Blackman (2006) have used common envelope models to map out different evolutionary routes in terms of angular momentum exchange. In 


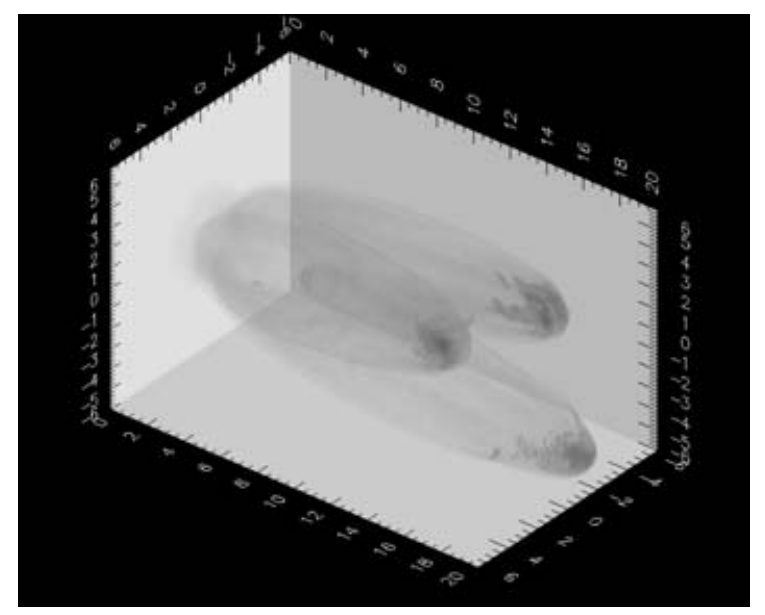

Figure 2. Simulation of 3 impulsively ejected hypersonic bullets as a model of CRL 618 . The image highlights the location of shocks as bullets propagate down the grid.

particular, they demonstrate how differential rotation profiles can be re-energized (from outside-in) via the influence of a companion. It appears then that so-called single star MHD models of outflow launching and collimation are untenable. The influence of a second, orbiting body is always required.

Observations appear to show that the majority of PNe begin life highly collimated with momentum too high to be driven by radiation pressure. Thus we turn to MRL models of PN outflow launching and collimation only to find that in all cases a companion is needed to provide conditions to create the field. In this way it appears that most PNe form from binaries. Our own perspective is that such a view should be a straw-man hypothesis to focus the efforts of the PNe community. Perhaps PNe should be seen as one of the variety of consequences of binary evolution. Obviously there are many questions which the hypothesis raises such as the already observed link between scale-heights and different morphological populations. Given the enormous importance of dynamos, MHD driven outflows and binary evolution across many fields of astronomy the presence of the issue in our community points the way to a grand challenge which we could attempt to solve.

\section{Acknowledgements}

This work was supported by Spitzer Space Telescope theory grant 051080-001, NSF grant AST-0406799 and DOE grant DE-F03-02NA00057.

\section{References}

Balick, B. \& Frank, A. 2002, AARA, 40, 439

Blackman, E. G., Frank, A., \& Welch, C. 2001a, ApJ, 546, 288

Blackman, E. G., Frank, A., Markiel, J. A., et al. 2001b, Nature, 409, 485

Bujarrabal, V., Castro-Carrizo, A., Alcolea, J., \& Sánchez Contreras, C. 2001, A\&A, 377, 868

Frank, A. \& Blackman, E. G. 2004, ApJ, 614, 737

Garcia-Segura, G., Langer, N., Rozyczka, M., \& Franco, J. 1999, ApJ, 517, 767

Matt, S., Frank, A., \& Blackman, E. G. 2006, ApJ, 647, L45

Nordhaus, J. \& Blackman, E. G. 2006, MNRAS, 370, 2004

Sahai, R. \& Trauger, J. T. 1998, AJ, 116, 1357

Soker, N. \& Rappaport, S. 2001, ApJ, 557, 256 